\title{
Finite Element Simulation of the Deformation of the Female Breast Based on MRI Data and 3-D Surface Scanning: An In-Vivo Method to Assess Biomechanical Material Parameter Sets
}

\author{
Stefan RAITH ${ }^{1}$, Maximilian EDER ${ }^{1}$, Fee von WALDENFELS ${ }^{1}$, Jalil JALALI ${ }^{2}$, Alexander VOLF $^{1}$, \\ Laszlo KOVACS ${ }^{1}$ \\ ${ }^{1}$ Research Group CAPS (Computer Aided Plastic Surgery) - Department of Plastic Surgery and Hand \\ Surgery, Klinikum rechts der Isar, Technische Universität München, Germany \\ ${ }^{2}$ Institute of Medical Engineering at the Technische Universität München (IMETUM), Garching, Germany
}

\begin{abstract}
Introduction:

Biomechanical studies of the mechanical deformations of the human body often use numerical simulations, such as the finite element analysis (FEA). Especially the shape changes of the female breast under varying load conditions are a current area of interest, both in the computational engineering science and the medical sector. During radiological diagnostics the breast is exposed to different mechanical loading conditions than later at the stage of the operation planning and in the operation room. For better operation planning, a prediction of these mechanical deformations on the computer is desired. However, to generate realistic results that consider the physics of biological materials, it is essential to have a sufficient understanding of the theoretical constitutive models and the material parameters that describe the soft tissue of the breast. Although numerous studies have been performed to acquire material parameters, yet no consensus of reliable parameter sets could be generated yet. We think that three-dimensional body scanning can have a decisive role for the determination of soft tissue parameters of the breast.
\end{abstract}

\section{Materials and Methods:}

In the presented study, twelve different parameter sets for material properties that have been proposed in literature references are employed to biomechanical simulation models. These 3-D anatomical models are derived from prone MRI datasets of 18 healthy volunteers. With the aid of FEA a force free reference state is calculated, using an iterative heuristic approach to overcome the deformations caused by unavoidable gravity loading. Starting from the obtained gravity free model the shape of the breast in upright position is calculated. The obtained result is then compared to the real volunteers' breast surfaces acquired with a 3-D surface scanner in order to evaluate the applicability of the simulation procedure.

\section{Results:}

It could be shown, that hyper-elastic constitutive models perform superior to linear elastic models that cannot exceed the linear Hookean domain. Within the group of hyper-elastic material models, proposed in literature, those found by Tanner at al. (2006) and Rajagopal at al. (2008), perform significantly $(p<0.01)$ better than the other material parameter sets evaluated. Variations in boundary conditions have shown a minor influence on the calculation outcome compared to the variation of material parameters.

\section{Conclusion:}

The advantage of the here presented method is its non-invasive character as a combination of volume imaging (MRI) and 3-D surface scanning (Laser triangulation) and the involvement of the computer for the actual simulation. Since the whole workflow of simulation and data evaluation is automated, multitudes of simulations can be performed with few additional efforts. Thus, optimizations of material parameters can be performed beyond the limits of parameter settings from previous studies that permit patient individual adjustment of material parameters. Thus, reliable biomechanical breast model based on the presented methods can be applied to derive patient specific material parameter sets. This data might be helpful in oncology for tumor tracking by integrating comparison of multimodality images into the simulation model and could improve plastic and reconstructive breast surgery planning.

Keywords: 3-D scan, MRI, Finite Element Simulation, material parameters, standardization 


\section{Introduction}

Surgical interventions due to breast cancer are very common surgical procedures in women with 140.337 cases in Germany in 2010 [1]. After breast ablatio it is often desired to reconstruct the amputated breast in order to regain symmetry and to imporve the life quality of the patients. These reconstructive surgeries are especially dificult due to the large soft tissue flaps that are necessary to reconstruct the missing breast with accurate volume and in the desired shape. Today, these operations are planned by drawing manually reference lines on the breast and the donor site. The success of the reconstruction thus mainly depends on the surgeons experiennce and skills. For the improvement of breast surgeries in this scope it is desired to have planning tools available that take advantage of modern measurement tools such as 3-D surface scanning and up to date simulation techniques such as the Finite Element Method. For these simulations, the mechanical properties of the human soft tissues are highly relevant. Only if the physical behavior of these structures can be modelled acuratelly, it is possible to plan relyably the operations in breast surgery.

Biomechanical studies of the mechanical deformations in the human body often use numerical simulations, such as the finite element analysis (FEA). Especially shape changes of the female breast under varying load conditions such as plain gravity or compression in mammography plates [2] are a current area of interest, both in the computational engineering science and the medical sector. During radiological diagnostics the breast is exposed to different mechanical loading conditions than later at the stage of the operation planning and in the operation room. For better operation planning, a prediction of these mechanical deformations with modern imaging and simulation techniques on the computer is desirable. However, to generate realistic results that consider the physics of biological materials, it is essential to have a sufficient understanding of the theoretical constitutive models and the material parameters that describe the soft tissue of the breast. Although numerous studies have been performed to acquire material parameters, yet no consensus of reliable parameter sets could be generated yet. We think that three-dimensional body scanning can have a decisive role for the determination of soft tissue parameters of the breast: In the presented study 3-D surface scanning is used in combination with volumetric Magnetic Resonance Imaging (MRI) to capture the breast shape in different positions. Simulations with the geometrical volume models from MRI are performed and the simulation results can be validated by using a comparison to 3-D surface scans. With this workflow it is possible to evaluate whether a certain material formulation is suitable for the simulation of the breast tissue.

\section{Material and Methods}

In the presented study, twelve different parameter sets for material properties that have been proposed in literature references are used on biomechanical simulation models. These 3-D anatomical models are derived from prone MRI datasets of 18 healthy volunteers (aged $26.9 \pm 3.0$ years with a range from 22 to 33). All volunteers gave their written informed consent to take part in the study and the Declaration of Helsinki protocols were strictly followed. Volunteers with known history of breast cancer or hereditary risk of breast cancer, acute breast infections, known autoimmune or infectious diseases, severe breast malformations and thoracic deformations or fibrocystic mastopathy and previous breast surgeries were excluded from the study. No indications of existing breast asymmetries were observed and no one of the volunteers did undergo any past surgical interventions in the breast area nor did they plan to do so in the future.

With the aid of FEA a force free reference state is calculated, using an iterative heuristic approach to overcome the deformations caused by unavoidable gravity loading. Starting from the obtained gravity free model the shape of the breast in upright position is calculated. The obtained result is then compared to the real volunteers' breast surfaces acquired with a 3-D surface scanner in order to evaluate the applicability of the simulation procedure.

\subsection{Volumetric Image Acquisition}

Volumetric Magnetic Resonance Imaging (MRI) data of the 18 volunteer was captured with the aid of a Philips Achieva 1.5 Tesla MRI scanner (Philips Medical Systems DMC GmbH, Hamburg, Germany) using a T1-weitghted imaging sequence with a $512 \times 512 \times 179$ voxel resolution and a spacing of 0.994 $\mathrm{mm} \times 0.994 \mathrm{~mm} \times 2 \mathrm{~mm}$ (imaging parameter: $4.6 \mathrm{~ms}$ ecco time and $9.2 \mathrm{~ms}$ repetition time). No intravenous contrast medium was applied. The thoracic images were obtained with the participants lying in prone position. The breasts did not touch the MRI bench. This was achieved with pillow supports located above the clavicle and in the shoulder region as well as caudal down to the lower belly area and the pelvic crest region. With this support structure, all compressions of the breast due to 
contact with the bench could be omitted. However the breast soft tissue is not stress-free because gravity forces still act. Thus the shape of the free hanging breast can be made available for further processing and segmentation in suitable imaging software packages. The resultant models can finally be used for Finite Element Simulations. But we have to keep in mind that these simulations do not start right away from an unloaded state, due to the acting gravitational forces. The different relevant anatomical compartements (see Fig. 1) have been segmented in a semi automiated procedure with the software tool Mimisc ${ }^{\circledR}$ (Materialise Inc., Leuven, Belgium) and exported as triangulated surfaces for the further processing and simulation model generation.
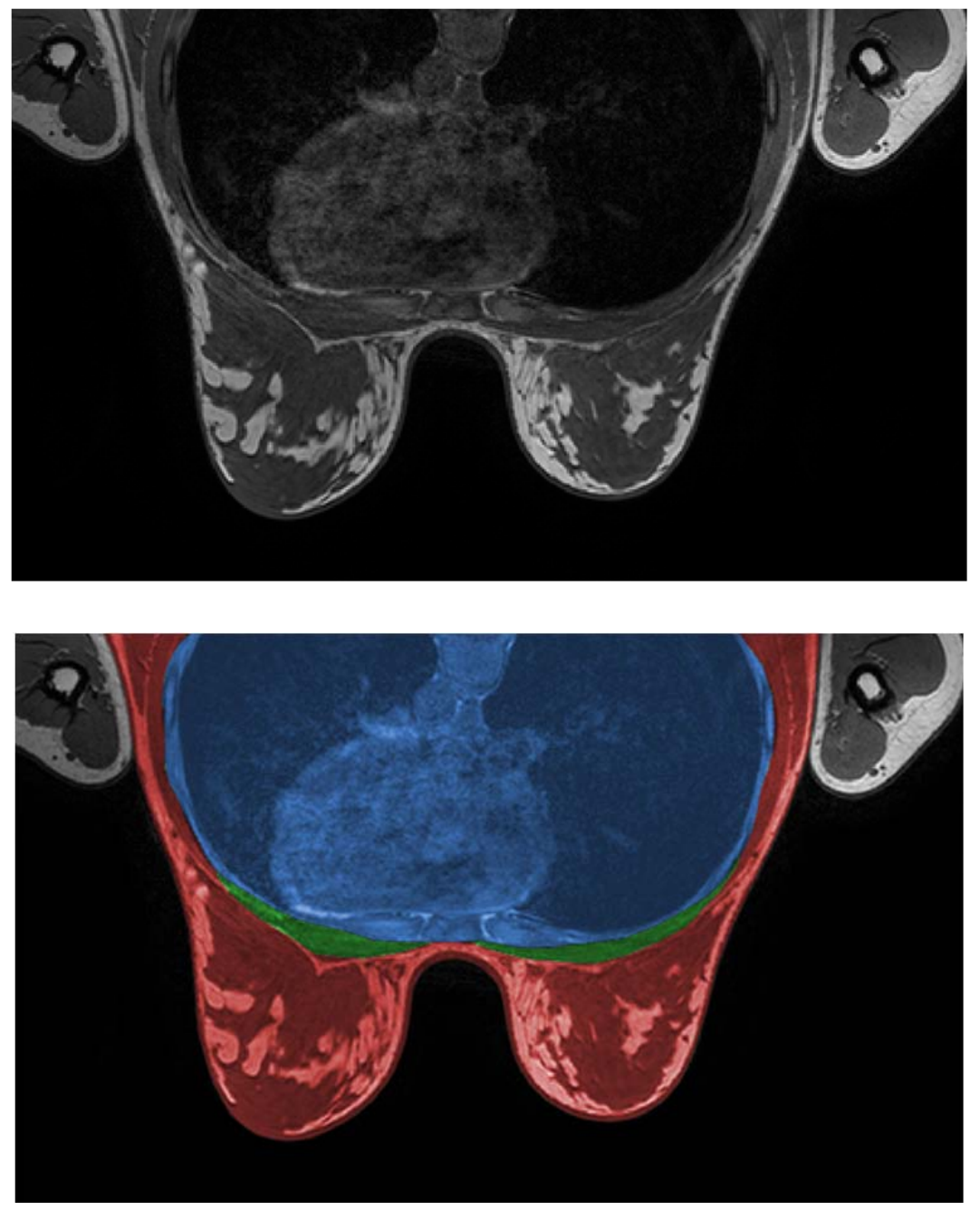

Fig. 1: MRI Images of the breast in prone position (top) and the segmentation of the relevant anatomical regions soft tissue, muscle tissue and thoracic wall (bottom).

\subsection{3-D Surface Scanning}

The post-operative outcome of a breast surgery with respect to symmetry is typically evaluated in standing position. However it is not yet common practice in clinical routine to use upright MRI systems due to its cost and the more difficult stable positioning of the patients. Usually in hospitals there are only MRI tube systems available that permit the image acquisition for patients in lying position. So the data for the internal anatomical structure is available either in prone or in supine positions. Three-dimensional surface scanning systems in contrast allow a variety of different positions of the patient including standing upright. Thus these techniques permit an indispensable advantage for the presented study.

The imaging in upright position was performed using a surface scanner working with laser triangulation (Konica-Minolta Co., Ltd., Osaka, Japan). This system has largely shown its applicability to breast shape measurements in preliminary studies [3] [4] [5] [6] [7]. The 3-D surface scans of the 18 participants were performed in standing position on predefined markers on the ground under 
standardized lighting conditions (light intensity 350-4000 lux) with a 10 degree upward angle of the scanner facing the participants $+30,0$ and -30 degrees relative to the lens in standing position [7]. During acquisition the females were asked to hold their breath. The arms have to be put down the side at the height of the pelvis and the back was supported by a wall to guarantee reproducible data by minimizing potential artifacts due to movements.

The acquired single shots from different angles (see Fig. 2 top) of each volunteer were converted into virtual 3-D models using appropriate software tools (Geomagic Studio $12^{\circledR}$, Raindrop Geomagic, Inc., NC, USA) that have already proven their applicability [3] [4] [5] [6] [7]. All potential problems for later work with the data such as holes due to insufficiently clear scanning data or intersections between different acquisitions were fixed. In Fig. 2 an overview of different surface models derived from 3-D laser scanning is given. For all models, the three single images (frontal, right $30^{\circ}$ and left $30^{\circ}$ ) could be merged into one representation of the full frontal part of the breast region. No holes or overlapping surface parts are present in the prepared models.
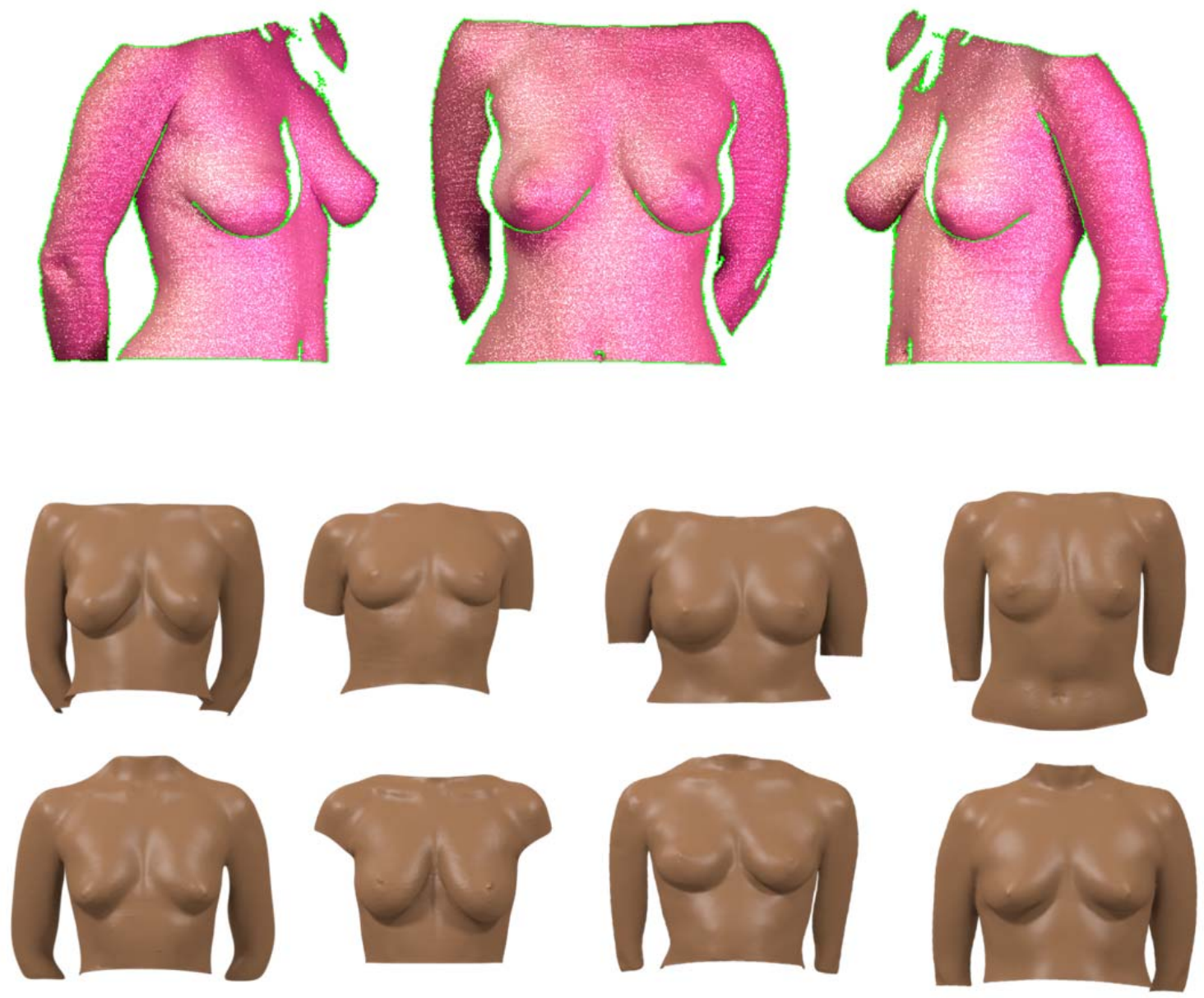

Fig. 2. Top: Three surface scans acquired with the Konica Minolta Vivid 911 device. The upright positions have been varied in 30 degrees to both sides in order to get the test person's side viewed surface information as well. These single shots have to be merged in a manual procedure using the software tool Geomagic. Surface scans of the breasts of eight exemplary test persons that participated in the study. Bottom: Eight representative surfaces processed with the described workflow. The anatomical variance in breast size and shape is obvious.

\subsection{Utilized material models}

Different material models are applied for the simulation of the female breast tissue: such as linear elastic, piecewise-linear elastic, exponential elastic and hyper-elastic constitutive models that have been proposed by several authors. Different methods of deriving the relevant parameters that describe the stiffness of the materials have been used: Krouskop et al. [8], Wellman et al. [9] and Samani et al. [10] obtained the necessary material parameter based on ex vivo indentation tests. Tanner et al. [2] 
used different material models in one publication including linear elastic, Neo-Hookean and Mooney-Rivlin hyper-elastic models according to different earlier publications [11] [10] [8].

Linear elastic material models, as used by Tanner et al. [12], do not permit enough deformation to describe the movement of the breast tissue due to gravity, thus these constitutive models have not been used in the presented study. Even if only gravity loading is applied, the strains exceed the Hookean domain of linear stress-strain relationship. Thus it is inevitable to use hyper-elastic material formulations to describe the deformations of the breast with finite element simulations.

In Fig. 3 an overview of different constitutive models proposed in literature is provided and their behavior for different load settings such as uniaxial, biaxial and shear loading. These models are compared in the presented study and their applicability for soft tissue simulation is investigated.

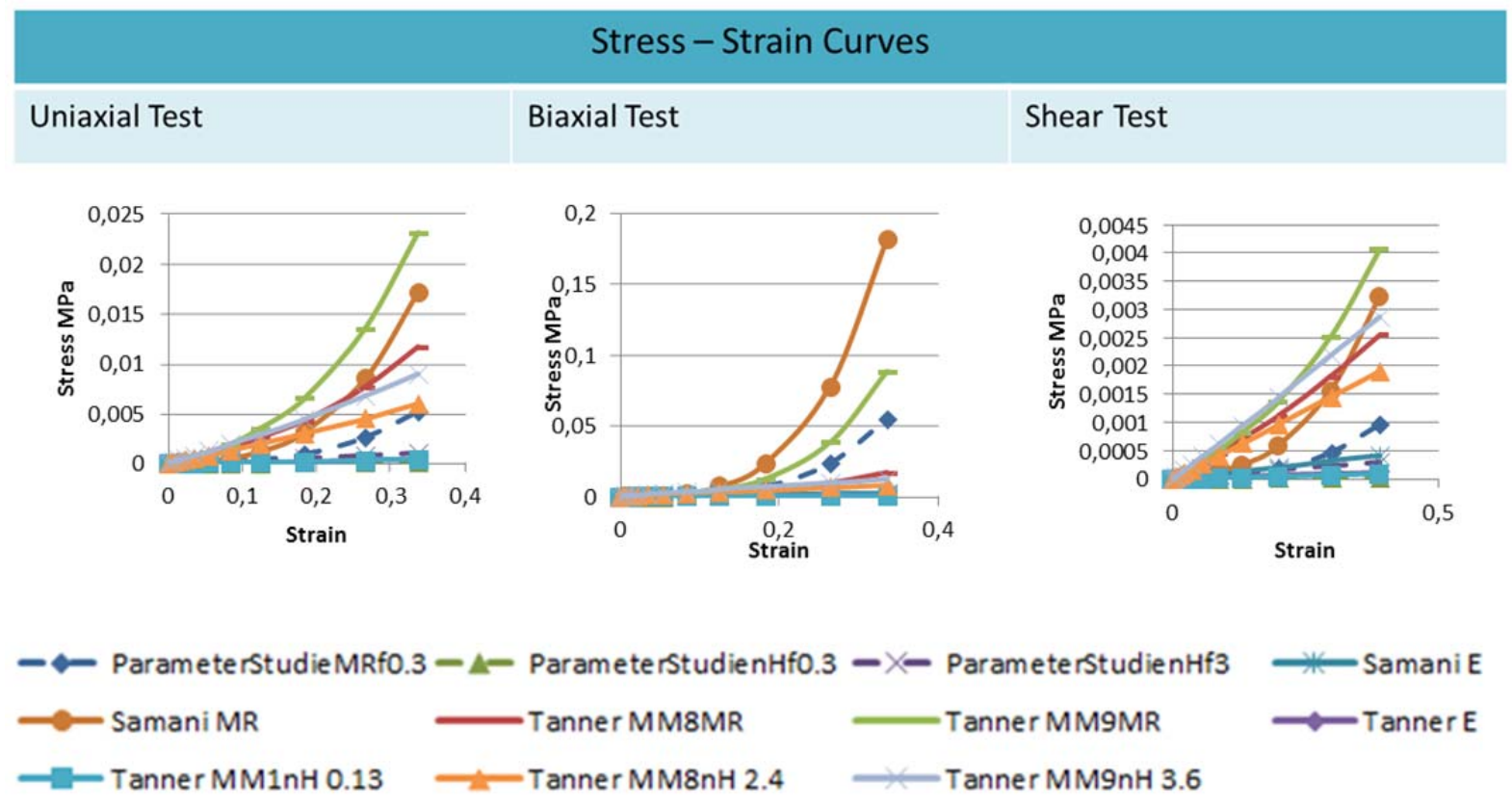

Fig. 3. Different behaviors of soft tissue models proposed by different literature references.

\subsection{Finite Element Simulation}

The segmented surfaces were processed in an adequate 3-D surface processing software (Geomagic Studio $12 \AA$, Raindrop Geomagic, Inc., NC, USA) to improve the surface quality and reduce segmentation artifacts that could hinder later mesh generation. For the finite element meshing the software ICEM (Ansys Inc. Canonsburg, PA, USA) has been applied.

Three surfaces containing the thoracic wall, pectoral muscle and the breast tissue as an entire component consisting of skin, fat and gland were used in ICEM to make a FEM volume mesh. Standardized demarcations of the models to the sides, top and bottom have been defined in order to guarantee inter-person comparability. Furthermore the element size has been chosen constant throughout all of the models for the same reason.

As mentioned above, the MRI data that is used to start with the simulation is derived from prone position. The gravity loading alone has an influence on the shape of the breast that is not negligible. Thus it is not correct to start with the simulations at this state. Instead a fist calculation has to be made to estimate the undeformed state of the breast. For this purpose a special algorithm has been developed to solve this task in an iterative loop (backward calculation). The obtained estimation of the undeformed configuration can be used to start a simulation (forward calculation) of a different position such as standing upright.

The programming language APDL (Ansys Parametric Design Language) was used for implementation and automatizing the whole process of Finite Element Simulation that has then ben performed with ANSYS (Ansys Inc. Canonsburg, PA, USA) [13]. 


\subsection{Comparison of simulations and 3-D surface scans}

The finite element simulation provides a breast geometry $n$ upright position. To determine the usability of a certain material parameter set for this type of calculation, a comparison with the real world has to be made (validation). For this purpose the result surface of the calculation as it is meshed in the finite element model is exported as a triangulated surface. This result can be compared to the 3-D laser scans of the breast shape. For accurate positioning of both models, landmarks such as the ones shown in Fig. 4 have been used.

For the 3-D comparisons a specially developed algorithm has been used that calculates the node to surface root mean square integration of the so called Haussdorff distance in $\mathrm{mm}$, according to the method described in [14]. The whole workflow is automated to allow fast processing of a lot of data with minimal efforts.
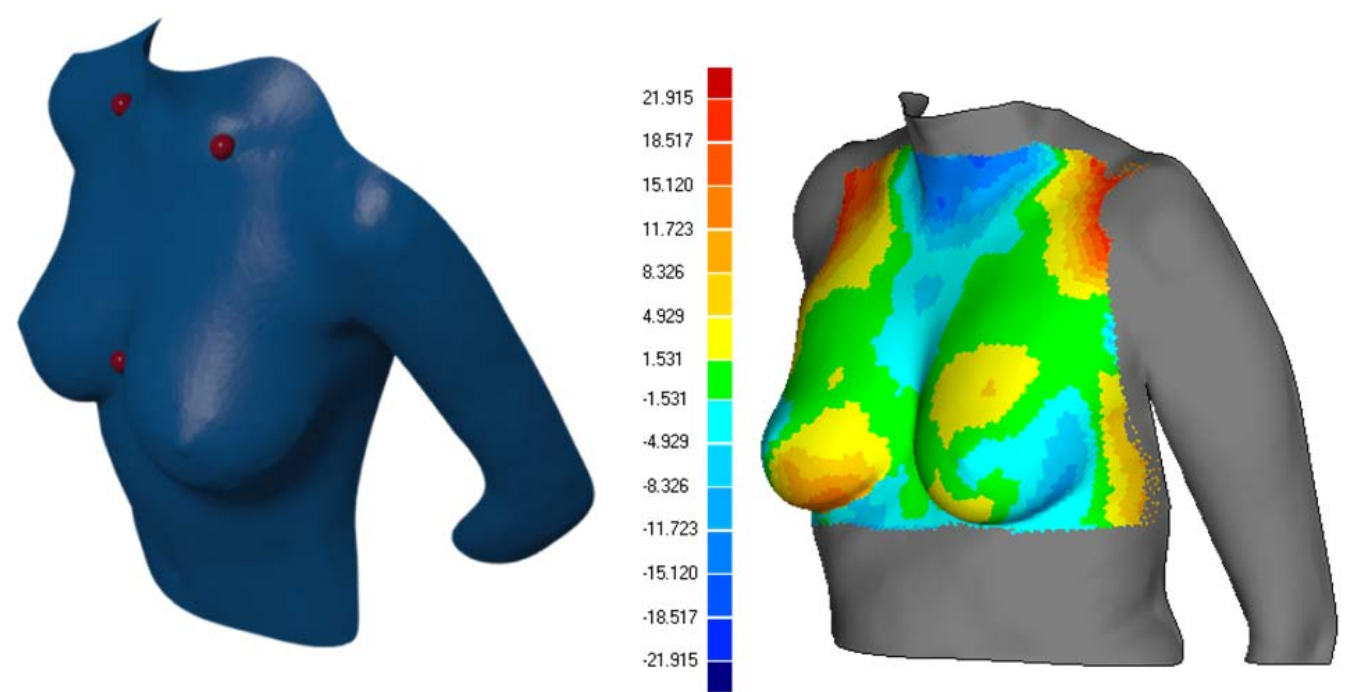

Fig. 4. 3-D surface scan (left) and comparison between FEM simulation result and scan (right).

\section{Results}

The applicability of the presented workflow for the simulation of the breast could be shown. The whole process is automated and thus permits an easy to use interface for the comparison of different material models with only minimal manual interactions.
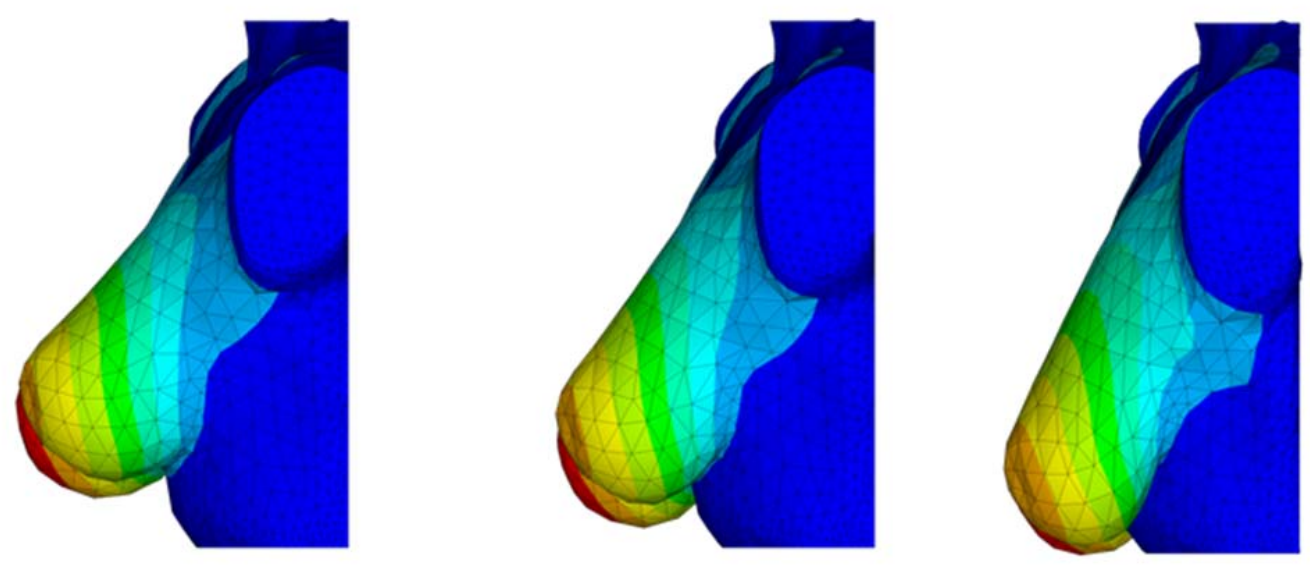

Fig. 5. Simulation results of the standing position with different material parameter sets. 
Due to the softness of the breast tissue it undergoes high deformations even at moderate loading conditions. Even gravity load alone is enough to exceed the linear Hookean domain. Thus the representation of the breast's soft tissue with purely linear elastic material models is not sufficient. Our finite element simulations did show a numerically instable behavior (divergence) when these material models have been applied.

Thus, it is inevitable to use nonlinear material models to be able to represent true stresses and strain in this tissue. Out of the numerous different material models that are proposed in literature, we took hyper-elastic models Neo-Hookean and Mooney-Rivlin materials that showed the best stability. However these material models showed big differences in stiffness as visualized in Fig. 5 for example. Many of the proposed materials show to stiff behavior and thus do not allow enough deformation as it is observed in the physiological study with the 3-D laser scanner.

Within the group of hyper-elastic material models that have been proposed in literature, those found by Tanner [2] and Rajagopal [15], perform significantly $(p<0.01)$ better than the other material parameter sets evaluated. These are relatively soft material data sets that allow high deformations due to only gravity loading. Variations in boundary conditions have shown a minor influence on the calculation outcome compared to the variation of material parameters.

\section{Conclusion}

The advantage of the here presented method is its non-invasive character as a combination of volume imaging (MRI) and 3-D surface scanning (Laser triangulation) and the involvement of the computer for the actual simulation. Since the whole workflow of simulation and data evaluation is automated, multitudes of simulations can be performed with few additional efforts. Thus, optimizations of material parameters can be performed beyond the limits of parameter settings from previous studies that permit patient individual adjustment of material parameters. Thus, reliable biomechanical breast models based on the presented methods can be applied to derive patient specific material parameter sets as well. This data might be helpful in oncology for tumor tracking by integrating comparison of multimodality images into the simulation model and could improve plastic and reconstructive breast surgery planning in the future.

\section{References}

[1] S. B. Deutschland, „Statistisches Bundesamt,“ [Online]. Available: https://www.destatis.de/DE/ZahlenFakten/GesellschaftStaat/Gesundheit/Krankenhaeuser/Tabell en/DiagnosenWeiblich.html. [Zugriff am 49 2012].

[2] C. Tanner, J. Schnabel, D. Hill und D. Hawkes, „Factors Influencing the Accuracy of Biomechanical Breast Models," Med Phys, Nr. 33, p. 1758-1769, 2006.

[3] M. Eder, F. Waldenfels, A. Swobodnik, M. Klöppel, A. Pape, T. Schuster, S. Raith, E. Kitzler, N. Papadopulos, H. Machens und L. Kovacs, „Objective breast symmetry evaluation using 3-D surface imaging," Breast, p. [Epub ahead of print], 2011.

[4] M. Eder, A. Schneider, H. Feussner, A. Zimmermann, C. Hoehnke, N. Papadopulos und E. Biemer, „Breast volume assessment based on 3D surface geometry: verification of the method using MR imaging,“ Biomed Tech, Bd. 53, pp. 112-121, 2008.

[5] L. Kovacs, M. Eder, R. Hollweck, A. Zimmermann, M. Settles, A. Schneider, M. Endlich, A. Mueller, K. Schwenzer-Zimmerer, N. Papadopulos und E. Biemer, „Comparison between breast volume measurement using 3D surface imaging and classical techniques," The Breast, Bd. 16, pp. 137-145, 2007.

[6] L. Kovacs, M. Eder, R. Hollweck, A. Zimmermann, M. Settles, A. Schneider, K. Udosic, K. Schwenzer-Zimmerer, N. Papadopulos und E. Biemer, „New Aspects of Breast Volume Measurement Using 3-Dimensional Surface Imaging,“ Ann Plast Surg, Bd. 57, pp. 602-610, 2006.

[7] L. Kovacs, A. Yassouridis, A. Zimmermann, G. Brockmann, A. Woehnl, M. Blaschke, M. Eder, K. Schwenzer-Zimmerer, R. Rosenberg, N. Papadopulos und E. Biemer, „Optimization of 3-dimensional imaging of the breast region with 3-dimensional laser scanners," Ann Plast Surg, Bd. 56, pp. 229-236, 2006. 
[8] T. Krouskop, T. Wheeler, K. Kallel, B. Garra und T. Hall, „Elastic moduli of breast and prostate tissues under compression," Ultrasonic Imaging, Nr. 20, p. 260-274, 1998.

[9] P. Wellman, R. Howe, E. Dalton und K. Kern, „Breast tissue stiffness in compression is correlated to histological diagnosis," Harvard University, 1999.

[10] A. Samani und D. Plewes, „A method to measure the hyperelastic parameters of ex vivo breast tissue samples," Phys Med Biol, Nr. 49, p. 4395-405, 2004.

[11] F. Azar, D. Metaxas und M. Schnall, „A deformable finite element model of the breast for predicting mechanical deformations under external perturbations," Acad Radiol, Bd. 8, p. 965975, 2001.

[12] C. Tanner, J. H. Hipwell und D. J. Hawkes, „Statistical deformation models of breast compressions from biomechanical simulations," Lecture Notes in Computer Science: International Workshop on Digital Mammography, pp. 426-432, 2008.

[13] Ansys Inc., Ansys Theory Manual v12.1.

[14] N. Aspert, D. Santa-Cruz und T. Ebrahimi, ,Mesh: Measuring Errors Between Surfaces Using the Hausdorff Distance," in IEEE International Conference in Multimedia and expo, Lausanne, 2002.

[15] V. Rajagopal, A. Lee, J. Chung, R. Warren, R. Highnam und M. Nash, "Creating individual-specific biomechanical models of the breast for medical image analysis.," Nr. 15, p. 1425-1436, 2008.

[16] G. Holzapfel, Nonlinear Solid Mechanics, Wiley, 2007.

[17] A. Lapuebla-Ferri, A. D. Palomar, J. Herrero und A.-J. Jimenez-Mocholi, „A patient-specific FE-based methodology to simulate prosthesis insertion during an augmentation mammoplasty," Med Eng Phys, Nr. 33, pp. 1094-1102, 2011.

[18] V. Rajagopal, P. M. F. Nielsen und M. P. Nash, „Modeling breast biomechanics for multi-modal image analysis - succeses and challenges," Systems Biology and Medicine, Bd. 2, Nr. 3, pp. 293-304, 2010.

[19] V. Rajagopal, J. Chung, D. Bullivant, P. Nielsen und M. Nash, „Finite elasticity: determining the reference state from a loaded configuration," International Journal of Numerical Methods in Engineering, Nr. 72, p. 1434-1451, 2007.

[20] A. Samani, J. Zubovits und D. Plewes, „Elastic Moduli of Normal and Pathological Human Breast Tissues:an inversion-technique-based investigation of 169 samples," Phys. Med Biol. 52, pp. 1565-1576, 62007.

[21] A. Samani, J. Bishop, M. Yaffe und D. Pelwes, „Biomechanical 3D finite element modeling of the Human Breast using MRI data," Nr. 20, pp. 271-279, IEEE Trans. Med. Imaging.

[22] A. Tregaskiss, P. Vermaak, R. Boulton und R. Morris, „The template technique for breast mound planning when using abdominal flpas for breast reconstruction," The Breast, pp. 1-4, 2012.

[23] A. Del Palomar, B. Calvo, J. Herrero, J. Lopez und D. M, „A finite element model to accurately predict real deformations of the breast," Medical Engineering and Physics, Bd. 30, p. 1089-1097, 2008.

[24] U. Ahcan, D. Bracun, K. Zivec, R. Pavlic und P. Butala, „The use of 3D laser imaging and a newe breast replica cast as a method to optimize autologous breast reconstruction after mastectomy," The Breast, Bd. 2, Nr. 21, pp. 183.-189, 2012. 